Research Article

\title{
Structural Health Monitoring of Timber Using Electromechanical Impedance (EMI) Technique
}

\author{
Fang Han $\mathbb{D},{ }^{1,2}$ Quanjing Zhang, ${ }^{1}$ Chengfeng Wang, ${ }^{1}$ Guangtao Lu, ${ }^{3}$ and Jinwei Jiang $\mathbb{D}{ }^{4}$ \\ ${ }^{1}$ College of Science, Wuhan University of Science and Technology, Wuhan 430065, China \\ ${ }^{2}$ Hubei Province Key Laboratory of Systems Science in Metallurgical Process, Wuhan University of Science and Technology, \\ Wuhan 430081, China \\ ${ }^{3}$ College of Mechanical Engineering and Automation, Wuhan University of Science and Technology, Wuhan 430065, China \\ ${ }^{4}$ Department of Mechanical Engineering, University of Houston, Houston, TX 77204, USA
}

Correspondence should be addressed to Fang Han; hanfang@wust.edu.cn and Jinwei Jiang; jjiang7@uh.edu

Received 20 November 2019; Revised 9 June 2020; Accepted 11 June 2020; Published 8 July 2020

Academic Editor: Ayman S. Mosallam

Copyright (c) 2020 Fang Han et al. This is an open access article distributed under the Creative Commons Attribution License, which permits unrestricted use, distribution, and reproduction in any medium, provided the original work is properly cited.

\begin{abstract}
Nowadays, the electromechanical impedance method has been widely used in the field of structural healthy monitoring, especially for the concrete and steel materials. However, the electromechanical impedance studies on damage detection for timber are limited due to the anisotropic and ununiform biomaterial properties. As a low-cost and environment-friendly building material, timber has been widely used in the construction. Thus, it is beneficial to develop electromechanical impedance technique for structural healthy monitoring of timber so as to ensure the stability and safety of the entire timber structures. In this paper, two damage factors, i.e., the damage location factor and the damage size factor of timber specimens are investigated by using the electromechanical impedance method. The method is implemented by using a patch of Lead Zirconate Titanate transducer both as an actuator to generate stress waves and a sensor to detect stress waves after propagating across the timber specimens. Then, the damage index-root mean square deviation is employed to evaluate the damage severity of the timber specimens. The results indicate that the damage index changes consistently with the change of damage location and size factors, and the proposed method using electromechanical impedance technique can efficiently estimate the damage and its severity.
\end{abstract}

\section{Introduction}

Timber, as a renewable, ubiquitous, and environmentfriendly material, is one of the popular construction materials in the long human history [1]. However, compared with metal and concrete, timber is prone to biological aggressions due to its organic nature. The damages caused by termite attacks and fungal decays will dramatically decrease the strength of the timber structures. In addition, some natural growth defects, such as knots, would also make timber as a nonhomogeneous material with initial holes and microcracks. Therefore, the damage of timber, whether it is congenital or acquired, if not detected at the early stage, can inevitably weaken the bearing capacity and shorten the service life span of the entire timber structures. Thus, the damage detection of timber structures has received increasing attention. A consensus is made that the monitoring of timber damage is essential to ensure the safety and reliability of the entire timber structures [2].

In recent decades, structural health monitoring (SHM) and damage detection techniques have advanced rapidly, various methods have been developed for timber structures, such as visual inspection, drill resistance, radiography, ultrasonics, vibration analysis, and stress waves [3]. For instance, Oh and Lee [4] conducted ultrasonic spectrum analysis with varied contact pressure to detect the small size damages such as insect damage in wooden cultural heritage. Osterloh et al. [5] achieved the high-quality images with $\mathrm{X}$-rays or gamma radiation to establish the location and extent of the timber damage. Choi et al. [6] proposed a modified damage index algorithm to locate the multiple damages and to evaluate the severity of timber damage, by 
normalizing the modal shape curvature and strain energy. However, generally speaking, complex equipment and algorithms are always needed for these methods. Therefore, the methods which are convenient, real time, and easy to implement are more welcomed in timber damage monitoring fields.

Recently, as a representative method of low cost, broadband frequency response, and dual functions of sensing and actuating, the SHM based on piezoelectric materials have been widely applied in different fields. One of the commonly used piezoelectric material is Lead Zirconate Titanate (PZT) $[7,8]$. PZT transducers are often used to generate and detect stress waves for damage detection in SHM [9-11]. Moreover, the PZT can be fabricated into various shapes to satisfy the special requirements across multiple industries [12-14]. The increasing reported literatures have showed that the SHM with PZT transducers are well applied in the fields of concrete crack detection $[15,16]$, metal pipeline corrosion detection $[17,18]$, bolt looseness monitoring $[19,20]$, and interlayer slide detection $[21,22]$.

Amongst the wildly used methods based on PZT materials are the active sensing method and the electromechanical impedance- (EMI-) based damage detection technique. Literatures have showed that the active sensing method has been well applied in the field of timber structures, for example, Zhang et al. $[23,24]$ applied the active sensing method to detect holes and cracks in timber structures and monitored the moisture content of timer specimens with the same method. Zhao et al. [25] monitored the bolt looseness in timer structures based on the time-reversal method with PZT transducers. Han et al. [26] applied the active sensing method to detect four common timber connection damages. Compared with active sensing method, the EMI method is more convenient since only one PZT patch is needed for a basic damage detection $[27,28]$. The EMI technique is initially presented by Liang et al. [29] to model the dynamic interaction between a host structure and a PZT transducer. Nowadays, the EMI technique has been widely used in the field of civil engineering [30-32], especially for the research on concrete strength monitoring [33-35] and damage detection [36]. However, to the best knowledge of the authors, the literatures about timber damage identification based on EMI are not as many as the traditional building materials. To some extent, the complexity of transmitted and reflected waves in different directions in timber has partially limited the application of EMI technique in wood structures [37]. Recently, Wang et al. [38] explored the feasibility of using the EMI technique for damage detection and proposed the Mahalanobis distance index based on root mean square deviation (RMSD) to evaluate the damage severity in timber specimens. Moreover, experimental studies need to be carried out to provide more detailed conclusions in the research field of timber structures.

In this paper, damage detection of timber specimens using EMI technique is studied. Both the damage location factor and damage size factor have been investigated to explore the sensitivity of electromechanical impedance signals. Then, the conventional damage index, RMSD is employed to evaluate the damage severity of the timber specimens. The results indicate that the structural health monitoring method using EMI technique for timber can estimate the damages efficiently in real time.

\section{Detection Principle}

2.1. Electromechanical Impedance. The EMI method is used to detect the common-hole damages of timber specimens by the PZT transducers. Recent research [39] has demonstrated that the PZT transducer can be employed both as an actuator and a sensor simultaneously due to its direct and inverse piezoelectric effects. The one-dimensional electromechanical impedance PZT-structure coupling system is shown in Figure 1. In this system, when an alternating current is applied on the PZT transducer, it will create harmonic vibrations with high frequencies, driving the vibrations of the host structure at the same time. Then, the electrical response is collected and analyzed in the form of electromechanical impedance. When damage occurs in the structure, the changed structural properties, such as the reduction of the stiffness and mass, will affect the electromechanical impedance of the PZT transducer accordingly. Therefore, by collecting and analyzing the difference of electromechanical impedance signals before and after the structural damages, the status of the damage in the structure can be detected in specific cases.

Nowadays, the widely used electromechanical model in the field of structure healthy monitoring is one-dimensional model which is first proposed by Zhang et al. [29]. However, considering the deformation of the PZT patch in other different directions, the 2D and 3D electromechanical models are also proposed. Recent research illustrates that $2 \mathrm{D}$ and 3D models are more complex and difficult to be applied in actual engineering projects. Thus, the one-dimensional model is more suitable for structural parameter identification and health monitoring. Therefore, the one-dimensional model without considering the influence of bonding layer in this paper is expressed as follows:

$$
\begin{aligned}
Y(\omega)= & j \omega \frac{\pi l_{a}^{2}}{4 h_{a}}\left\{\bar{\varepsilon}_{33}^{T}-d_{31}^{2} \bar{Y}^{E}+\left(\frac{Z_{a}(\omega)}{Z_{s}(\omega)+Z_{a}(\omega)}\right) d_{31}^{2} \bar{Y}^{E}\right. \\
& \left.\cdot\left(\frac{\tan k l_{a}}{k l_{a}}\right)\right\},
\end{aligned}
$$

where $\omega$ denotes the angular frequency under driving voltage, $Y(\omega)$ is the electromechanical admittance (reciprocal of impedance $Z(\omega))$ of PZT patches at angular frequency $\omega$, $Z_{a}(\omega)$ and $Z_{s}(\omega)$ represent the mechanical impedance of PZT paths and host structure at angular frequency $\omega$, respectively, $l_{a}$ and $h_{a}$ denote the diameter and thickness of PZT patches, respectively, $\bar{\varepsilon}_{33}^{T}$ is the complex electric permittivity when the stress is equal to zero, $d_{31}$ is the piezoelectric constant of the PZT patch, $\bar{Y}^{E}$ is the complex Young's modulus of PZT patch when the electric field is equal to zero, $k$ is the wave number, and $j$ is the imaginary unit.

As indicated in equation (1), if the properties of PZT patches remain unchanged with environment, the output signal of electromechanical admittance $Y(\omega)$ for the 


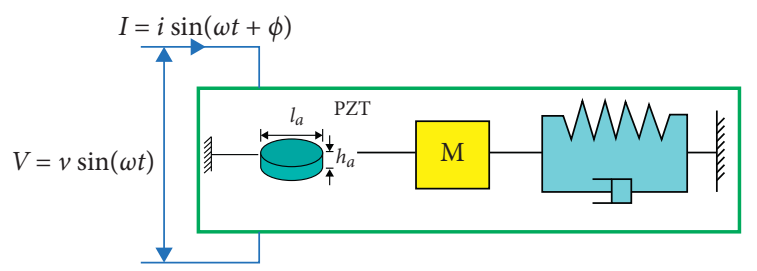

Figure 1: The one-dimensional electromechanical impedance PZT-structure coupling system.

electromechanical coupling system uniquely depends on the mechanical impedance of the host structure. Therefore, the change of PZT electrical impedance can reflect the change of mechanical impedance of the host structure accordingly when a damage occurs. This is the principle for detecting the damages of timber specimens based on the EMI technique. Park and Inman [40] have shown that the impedance-based method can identify local, minor changes in structural integrity at a very early stage since high frequency responses are very sensitive to changes in the structural integrity.

2.2. Statistical Damage Indices. In order to evaluate the damage severity of timber specimens quantitatively and accurately, the conventional damage index, root-meansquare deviation (RMSD) is used to analyze the changes of PZT electromechanical impedance signatures. The mathematical expression of the RMSD is expressed as follows:

$$
\operatorname{RMSD}=\sqrt{\frac{\sum_{i=1}^{N}\left\{\operatorname{Re}\left(Z_{i, 1}\right)-\operatorname{Re}\left(Z_{i, 0}\right)\right\}^{2}}{\sum_{i=1}^{N}\left\{\operatorname{Re}\left(Z_{i, 0}\right)\right\}^{2}}} \times 100 \%,
$$

where Re denotes the real part of the electromechanical impedance, $Z_{i, 0}$ is the impedance of the PZT patch measured in the initial intact state, $Z_{i, 1}$ is the impedance of the PZT patch measured in the damaged state, and $N$ represents the number of the frequency points. For the undamaged structure, the RMSD value is very close to 0 . For several cases, when the severity of damage is further increased, the RMSD value will change accordingly. Thus, the value of RMSD can effectively reflect the damage severity of the timber specimens.

\section{Experimental Setup}

3.1. Timber Specimen. To verify the proposed theory, five groups of timber specimens (pine wood from North America) with the same dimensions $(200 \mathrm{~mm} \times 60 \mathrm{~mm} \times 17 \mathrm{~mm})$ are used in this experiment. All the timber samples are cut down from a single piece of wood and have no obvious visual flaws, so they have almost the same natural properties to reduce the material errors. For each group, one circular PZT patch (diameter $=10 \mathrm{~mm}$ and thickness $=2 \mathrm{~mm}$ ), which are sandwiched structures with two electrode layers and one layer of PZT, is mounted at the predetermined location $(30 \mathrm{~mm}$ to the left side) using epoxy (Loctite Heavy Duty 5 min epoxy). To mimic the common damages of timber structures in daily life, different diameters of artificial holes, from small to large, are drilled in all the timber specimens on the workbench, as shown in Figure 2.

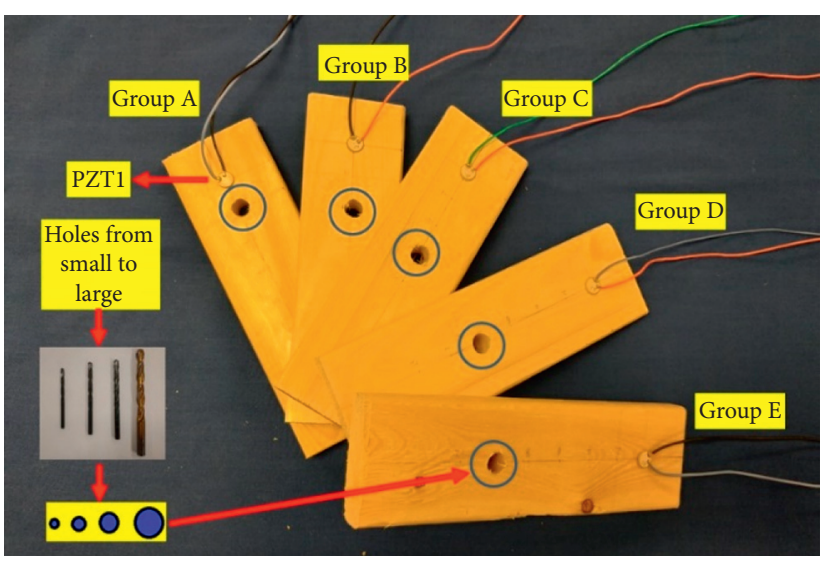

Figure 2: Timber specimens with holes.

In this study, different damage cases with various damage locations and damage sizes are investigated. The diameter of the artificial hole, which is denoted as $\phi$, varies over five different sizes: $0,4,6,8$, and $11 \mathrm{~mm}$, respectively. The distance between the artificial hole and the PZT transducer, which is denoted as $L$, varies over five different sizes: $2,4,6,8$, and $10 \mathrm{~mm}$, respectively. Moreover, the depth of the artificial holes are the same as the depth of the timber specimens. Therefore, a total of 25 different cases are considered in this study. The details of the specimens of Groups A, B, C, D, and E are tabulated in Table 1. The name of test cases are abbreviated as "Case number-Group number" in the following analysis. For example, Case 3-D means the damaged Case 3 in Group D with diameter $\phi=6 \mathrm{~mm}$ and location $L=80 \mathrm{~mm}$. The schematic of different timber specimens with damages are shown in Figure 3.

\subsection{Experimental Setup and Experimental Procedure. The} experimental apparatus includes a data acquisition system (the Agilent 4294A, Keysight Technologies), a laptop with the acquisition software, and timber specimens with PZT patches, as depicted in Figure 2. The laptop and the impedance analyzer are connected with a LAN cable. The schematic of damage detection of timber specimen based on EMI technique is shown in Figure 4. Moreover, all the timber specimens are fixed on the same bench clamp with the same clamping position. It is also worth noting that the number of turns of handle must be kept the same when torque are applied to all the timber specimens in order to minimize the boundary influence of the environment. Then, the artificial hole damage is performed by the hand drill with four different diameters of drills. The total experimental apparatus is shown in Figure 5.

First of all, a sweeping frequency band ranged from $100 \mathrm{kHz}$ to $1 \mathrm{MHz}$ is performed on the bonded PZT transducer to determine the optimal frequency band of the electromechanical coupled system in the test. The max number of the EMI signature spectra, totally 801 sampling points are selected to ensure the accuracy of the acquired signature. Both the real part of the impedance spectra (resistance) and the imaginary part of the impedance spectra 
TABLE 1: Test cases of timber damage in Groups A, B, C, D, and E.

\begin{tabular}{lcccccccccc}
\hline & \multicolumn{2}{c}{ Intact case } & \multicolumn{2}{c}{ Damaged cases } \\
Group & \multicolumn{2}{c}{ Case 1 } & \multicolumn{2}{c}{ Case 2 } & \multicolumn{2}{c}{ Case 3 } & \multicolumn{2}{c}{ Case 4} & Case 5 \\
& $\phi(\mathrm{mm})$ & $L(\mathrm{~mm})$ & $\phi(\mathrm{mm})$ & $L(\mathrm{~mm})$ & $\phi(\mathrm{mm})$ & $L(\mathrm{~mm})$ & $\phi(\mathrm{mm})$ & $L(\mathrm{~mm})$ & $\phi(\mathrm{mm})$ & $L(\mathrm{~mm})$ \\
\hline Group A & 0 & 0 & 4 & 20 & 6 & 20 & 8 & 20 & 11 & 20 \\
Group B & 0 & 0 & 4 & 40 & 6 & 40 & 8 & 40 & 11 & 40 \\
Group C & 0 & 0 & 4 & 60 & 6 & 60 & 8 & 60 & 11 & 60 \\
Group D & 0 & 0 & 4 & 80 & 6 & 80 & 8 & 80 & 11 & 80 \\
Group E & 0 & 0 & 4 & 100 & 6 & 100 & 8 & 100 & 11 \\
\hline
\end{tabular}

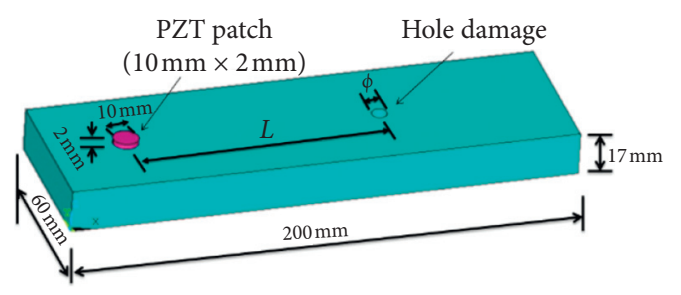

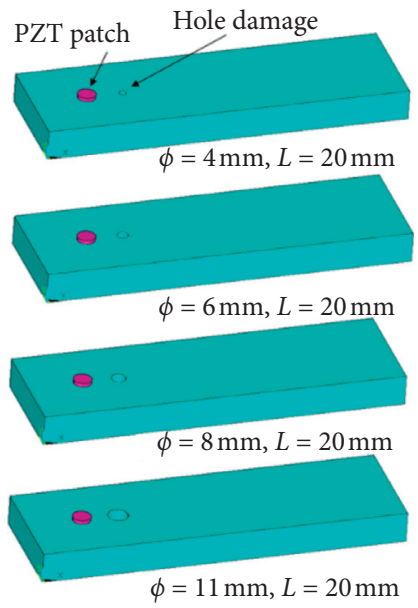

(b)

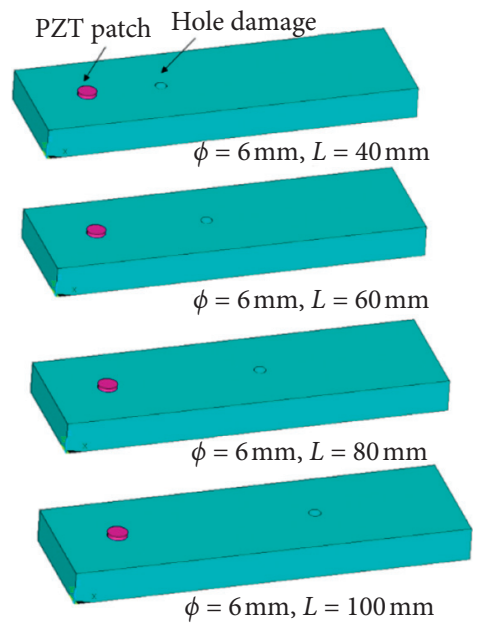

(c)

Figure 3: The schematic of different timber specimens with different damages. (a) The dimension of the timber specimen. (b) Holes with a fixed position and varied diameters. (c) Holes with a fixed diameter and varied locations.

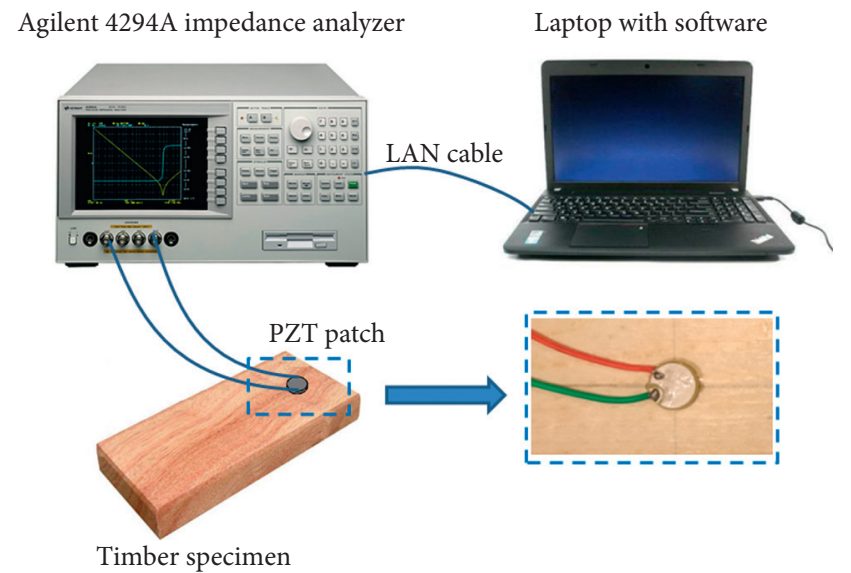

FIgURE 4: The schematic of damage detection of timber specimen based on EMI technique.

(reactance) are measured for further analysis. Then, the frequency band from $100 \mathrm{kHz}$ to $1 \mathrm{MHz}$ is selected to measure both the resistance and reactance signatures at the intact case of the timber specimens. The curves of the resistance and reactance with frequency are shown in Figures 6(a) and 6(b), respectively. Both of the figures show that there are two main observed resonant frequency peaks (RFP) at the frequency range from $150 \mathrm{kHz}$ to $550 \mathrm{kHz}$

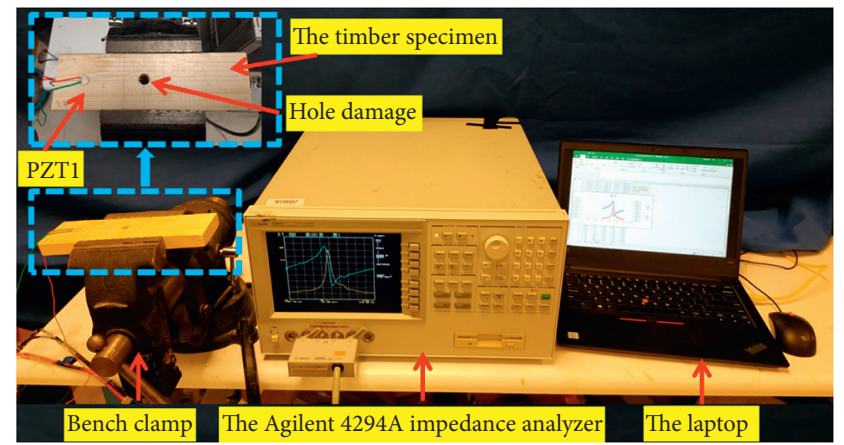

FIGURE 5: Experimental setup.

approximately. More specifically, the first RFP with the larger magnitude is at the frequency range from $150 \mathrm{kHz}$ to $300 \mathrm{kHz}$ and the second RFP with the smaller magnitude is at the frequency range from $450 \mathrm{kHz}$ to $550 \mathrm{kHz}$. It is obvious that the magnitude of both the resistance and reactance at the first RFP are much larger than that at the second RFP, and it is almost tenfold as the second one. Therefore, the frequency band from $150 \mathrm{kHz}$ to $300 \mathrm{kHz}$ is selected for further analysis. Park and Inman [40] have shown that the resistance signature is more sensitive and stable than the reactance signature to the changes of structural mechanical properties, and therefore only the real part of the impedance 


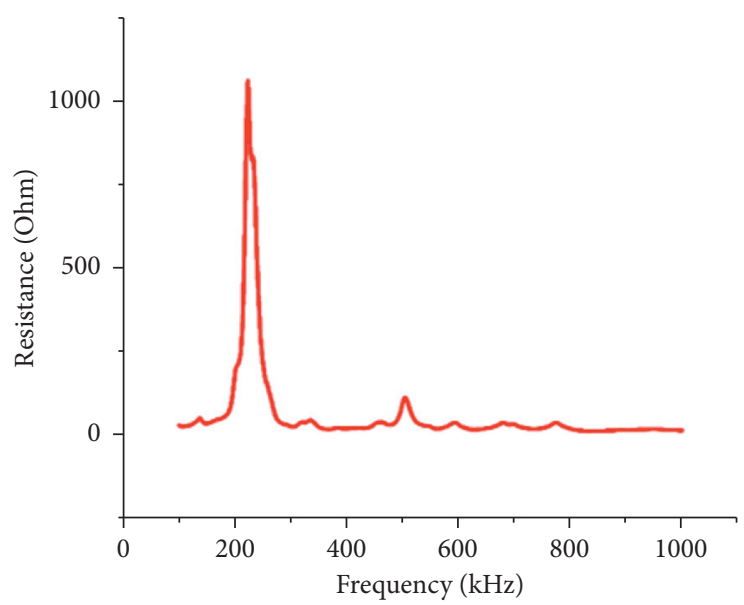

(a)

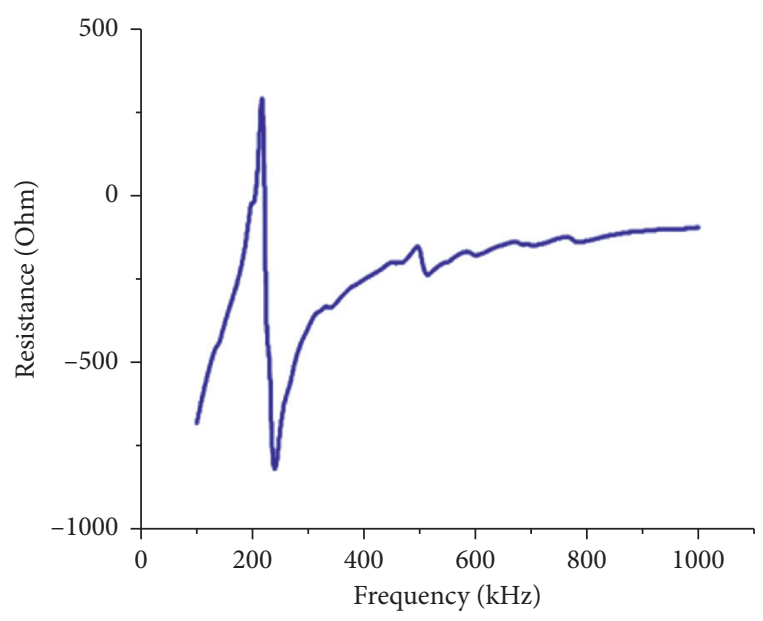

(b)

Figure 6: Resistance and reactance signatures at frequency range from $100 \mathrm{kHz}$ to $1 \mathrm{MHz}$. (a) The relationship between resistance and frequency. (b) The relationship between reactance and frequency.

(resistance) in the frequency band ranged from $150 \mathrm{kHz}$ to $300 \mathrm{kHz}$ is selected as the measured signature in the following study. Furthermore, each case from intact to damaged is measured five times, and thus the average of five measurements is calculated to reduce the measurement errors. At last, it is worth mentioning that all the tests should be finished within two hours in the laboratory to minimize the influence of the environmental humidity on the results, and in this case the humidity and temperature change during the tests could be ignored.

\section{Results and Discussion}

4.1. Impedance Signatures. Figure 7 shows the impedance signatures measured in different groups from $150 \mathrm{kHz}$ to $300 \mathrm{kHz}$. From these figures, it can be found that the resonant frequency and the impedance magnitude maintain similar trends under different damage status. Specifically, the resonant frequency corresponding to the peak impedance is about $250 \mathrm{kHz}$. In addition, the peak impedance decreases with the increase of the damage severity. In other words, the intact status (Case 1 in every group) has the largest peak resistance, and the value under the damage status (i.e., Case 2, Case 3, Case 4, and Case 5 in every group) decreases sequentially. Moreover, the first group (Group A), namely, $L=20 \mathrm{~mm}$ case, shows the most obvious tendency among five different groups, and the next is the Group B, i.e., $L=40 \mathrm{~mm}$. The change of the other three groups are not as obvious as that of the first two groups. Additionally, it is worth noting that the peak impedance curves appear as a very slight shift in enlarged figures from Group A to Group E, respectively. This phenomenon can be explained through the changes of structural stiffness under different damage cases. In other words, the closer the PZT transducer to the damage hole is, the more obvious the measured frequency changes. Therefore, the EMI technique can detect the damage status of the timber specimens.
In order to evaluate the severity of damage more quantitatively and accurately, the conventional damage index, root-mean-square deviation (RMSD) expressed as equation (2), is calculated to analyze the changes of impedance signatures.

4.2. Damage Index. Figure 8(a) shows the variation of damage index RMSD with different damaged cases. It can be seen obviously from Figure $8(\mathrm{~b})$ that when the damage size (diameter of the hole) is fixed, the RMSD decreases gradually with the increase of the distance between the PTZ transducer and damage location. For example, when the diameter $\phi=8 \mathrm{~mm}$ (Case 4), the RMSD decreases from $7.01 \%(L=20 \mathrm{~mm})$ to $6.22 \%(L=40 \mathrm{~mm}), 4.38 \%(L=60 \mathrm{~mm})$, $3.61 \%(L=80 \mathrm{~mm})$, and $3.26 \%(L=100 \mathrm{~mm})$, respectively. A larger RMSD value indicates a more closer distance from the PTZ transducer to the damage location; in other words, the PZT transducers are more sensitive in detecting local damage in timber specimens (especially for Group A,B). After that, the RMSD index still goes down but with a gradually decreasing rate (especially for Group C, D, and E), as shown in Figure 8(b). The RMSD value tends to be saturated when the hole is far away from the PZT transducer.

On the contrary, when the damage location (distance between the PTZ transducer and hole) is fixed, the RMSD increases gradually with the increase of the hole diameter, as shown in Figure $8(\mathrm{c})$. For example, when the distance between the PZT transducer and the hole is $20 \mathrm{~mm}$, i.e., $L=20 \mathrm{~mm}$ (Group A), the RMSD increases from $5.53 \%(\phi=4 \mathrm{~mm})$ to $6.60 \%(\phi=6 \mathrm{~mm})$, $7.01 \%(\phi=8 \mathrm{~mm})$, and $9.40 \%(\phi=11 \mathrm{~mm})$, respectively. A larger RMSD value indicates a more severe damage signal detected in the timber specimens. Moreover, another variation tendency is that the RMSD index goes up rapidly when the PZT transducer is closer to the damage hole (especially for Groups A and B). With the increasement of the distance, the RMSD index still goes up but with a slow increasing rate (especially for Groups C, D, and E), as shown in Figure 8(c). 

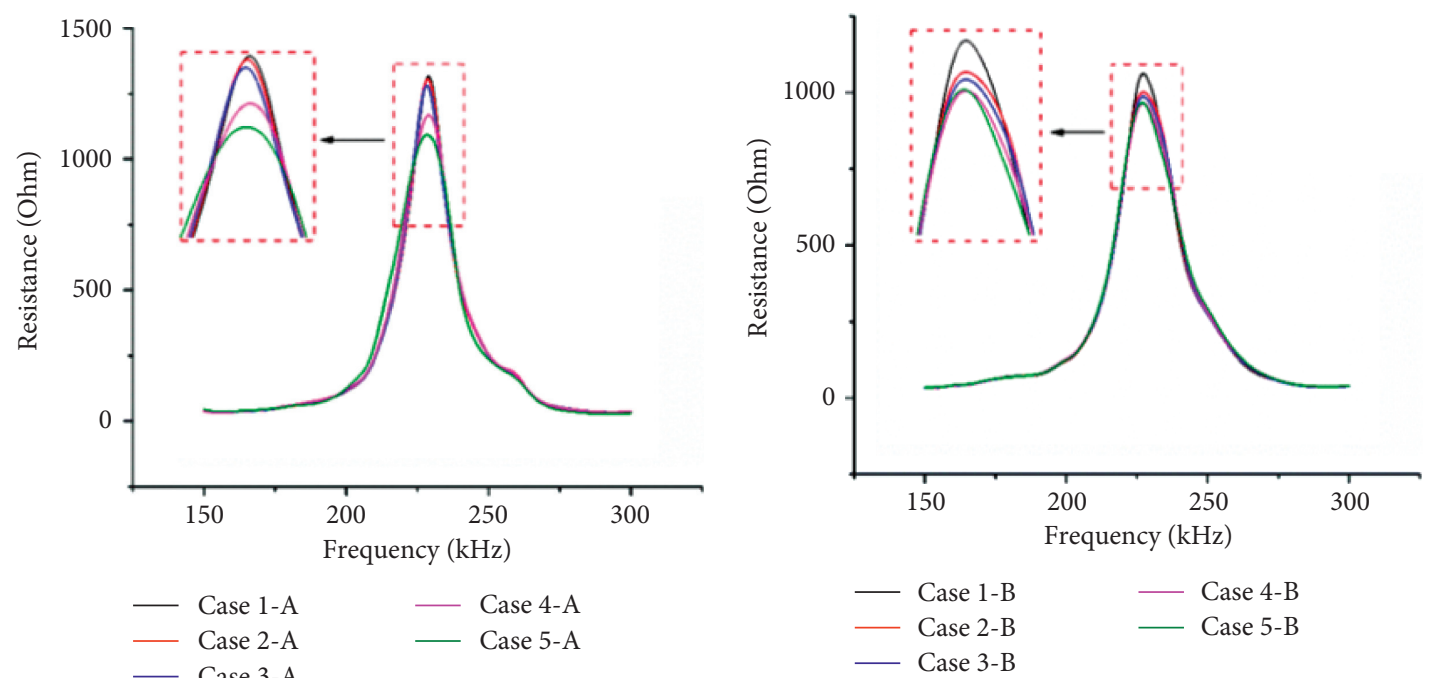

(a)

(b)
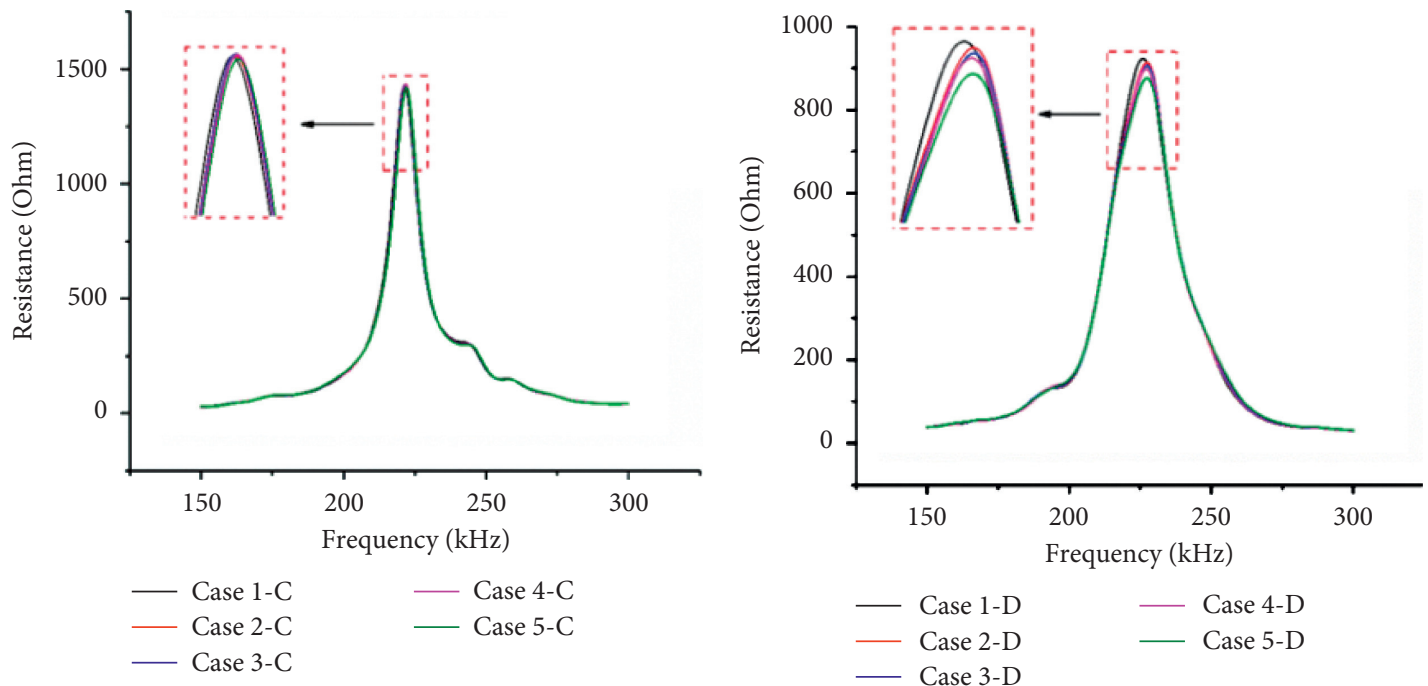

(c)

(d)

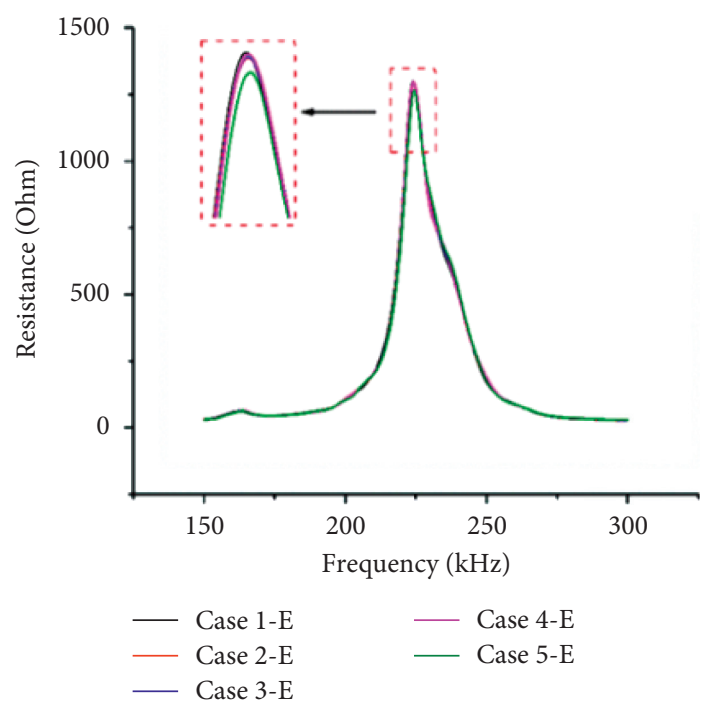

(e)

Figure 7: Impedance signatures in different cases from $150 \mathrm{kHz}$ to $300 \mathrm{kHz}$. (a) Cases 1-5 in Group A; (b) Cases 1-5 in Group B; (c) Cases 1-5 in Group C; (d) Cases 1-5 in Group D; (e) Cases 1-5 in Group E. 


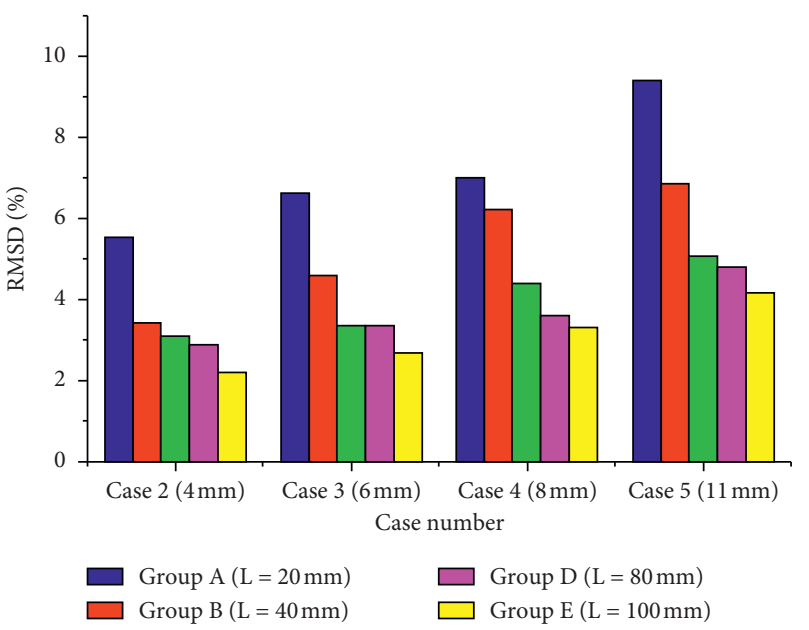

(a)

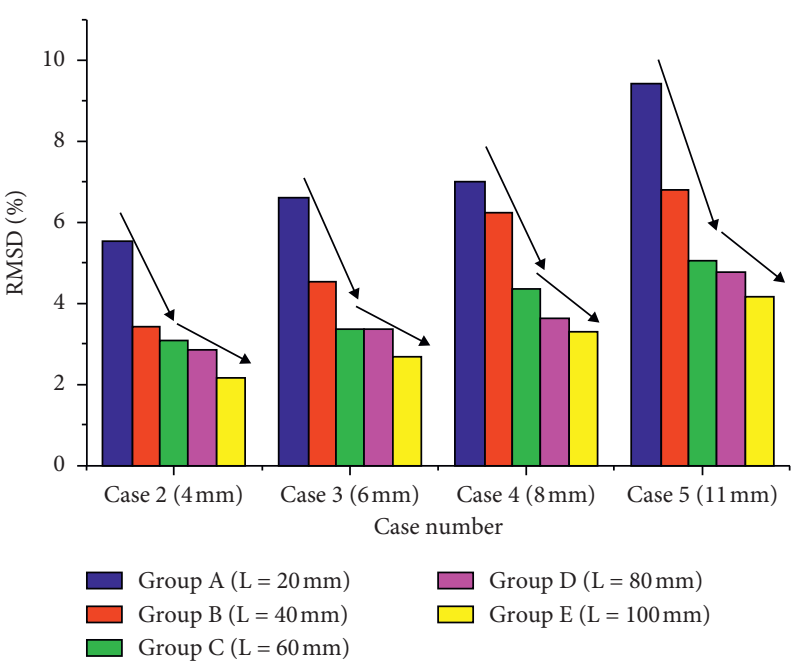

(b)

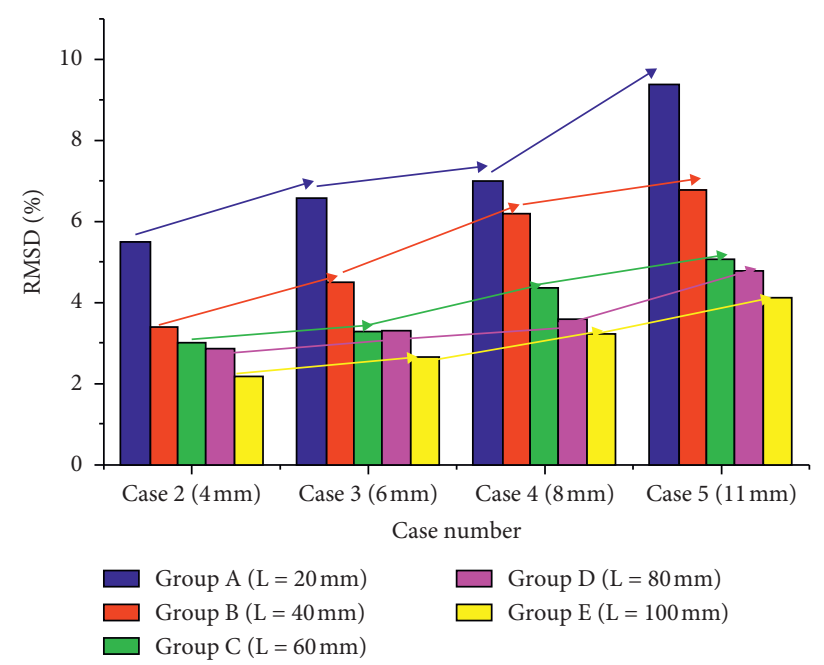

(c)

FIGURE 8: The variation of damage index RMSD with different damaged cases. (a)The variation of RMSD in different cases. (b) The variation tendency of RMSD with damage location. (c)The variation tendency of RMSD with damage size.

\section{Conclusions and Future Work}

In this paper, two damage factors, the damage location and damage size of timber specimens are investigated by using the EMI technique. Then, the RMSD-based damage index is proposed to evaluate the damage severity quantitatively. The experimental results show that the EMI technique is effective and sensitive to the local damage of the timber specimens, especially in the frequency range from $150 \mathrm{kHz}$ to $300 \mathrm{kHz}$. Particularly, when the damage size is fixed, the RMSD index decreases gradually with the increase of the distance between the PTZ transducer and damage location. On the contrary, when the damage location is fixed, the RMSD increases gradually with the increase of damage size. The technique based on EMI can effectively detect the damages of the timber specimens in real time.

However, it is also worth noting that the results are more sensitive to the co-location of the sensor and the damage. So, further studies are urgently needed to investigate the "valid detection distance" on larger timber specimens in the future work. In addition, future work will also involve considering more factors affecting the timber damages, such as different damage types (i.e., crack or hole), different damage distributions (i.e., scatter or continuous), and different experimental conditions (i.e., temperatures, humidity, and boundary). Then, the coupling effects of multiple damage factors will be studied and classified based on the EMI technique in real-time practice.

\section{Data Availability}

The data used to support the findings of this study are available from the corresponding author upon request.

\section{Conflicts of Interest}

The authors declare that they have no conflicts of interest. 


\section{Acknowledgments}

The research was partially supported by Hubei Province Key Laboratory of Systems Science in Metallurgical Process (Wuhan University of Science and Technology, Grant no.Y201904). The authors are also grateful for the financial support from National Natural Science Foundation of China (Grant nos. 51108358 and 51574184).

\section{References}

[1] C. Helena, Y. David, and T. Eleftheria, "Guidelines for on-site assessment of historic timber structures," International Journal of Architectural Heritage, vol. 9, no. 3, pp. 277-289, 2015.

[2] H. Liu, C. Koyama, J. Zhu, Q. Liu, and M. Sato, “-Earthquake damage inspection of wood-frame buildings by a polarimetric GB-SAR system," Remote Sensing, vol. 8, no. 11, pp. 935-945, 2016.

[3] E. Liu, D. Pollock, and J. Kainz, "Nondestructive evaluation techniques for timber bridges," in Proceedings of the 5th World Conference on Timber Engineering, Montreaux, Switzerland, 1998.

[4] J.-K. Oh and J.-J. Lee, "Feasibility of ultrasonic spectral analysis for detecting insect damage in wooden cultural heritage," Journal of Wood Science, vol. 60, no. 1, pp. 21-29, 2014.

[5] K. Osterloh, C. Raedel, U. Zscherpel et al., "Fast neutron radiography and tomography of wood," Insight-Non-destructive Testing and Condition Monitoring, vol. 50, no. 6, pp. 307-311, 2008.

[6] F. C. Choi, J. Li, B. Samali, and K. Crews, "Application of modal-based damage-detection method to locate and evaluate damage in timber beams," Journal of Wood Science, vol. 53, no. 5, pp. 394-400, 2007.

[7] G. Wang, "Analysis of bimorph piezoelectric beam energy harvesters using timoshenko and euler-bernoulli beam theory," Journal of Intelligent Material Systems and Structures, vol. 24, no. 2, pp. 226-239, 2013.

[8] I. Kurt, S. D. Akbarov, and S. Sezer, "The influence of the initial stresses on lamb wave dispersion in pre-stressed PZT/ Metal/PZT sandwich plates," Structural Engineering and Mechanics, vol. 58, no. 2, pp. 347-378, 2016.

[9] J. Li, H. Hao, Y. Xia, and H.-P. Zhu, "Damage detection of shear connectors in bridge structures with transmissibility in frequency domain," International Journal of Structural Stability and Dynamics, vol. 14, no. 02, p. 1350061, 2014.

[10] Y. Wang, X. Q. Zhu, H. Hao, and J. P. Ou, "Guided wave propagation and spectral element method for debonding damage assessment in RC structures," Journal of Sound and Vibration, vol. 324, no. 3-5, pp. 751-772, 2009.

[11] F. Wang and G. Song, "Bolt early looseness monitoring using modified vibro-acoustic modulation by time-reversal," Mechanical Systems and Signal Processing, vol. 130, pp. 349-360, 2019.

[12] W. Liao and C. Chiu, "Seismic health monitoring of a space reinforced concrete frame structure using piezoceramic-based sensors," Journal of Aerospace Engineering, vol. 32, no. 3, Article ID 04019015, 2019.

[13] L. S. Huo, D. D. Chen, Y. B. Liang, H. N. Li, X. Feng, and G. B. Song, "Impedance based bolt pre-load monitoring using piezoceramic smart washer," Smart Materials and Structures, vol. 26, no. 5, Article ID 057004, 2017.
[14] J. Wu, Q. Kong, I. Lim, and G. Song, "Feasibility study of interlayer slide monitoring using postembedded piezoceramic smart aggregates," Journal of Sensors, vol. 2018, pp. 1-10, 2018.

[15] Q. Kong, Q. Feng, and G. B. Song, "Water presence detection in a concrete crack using smart aggregates," International Journal of Smart and Nano Materials, vol. 6, no. 3, pp. 149161, 2015.

[16] Q. Kong, R. Robert, P. Silva, and Y. Mo, "Cyclic crack monitoring of a reinforced concrete column under simulated pseudo-dynamic loading using piezoceramic-based smart aggregates," Applied Sciences, vol. 6, no. 11, p. 341, 2016.

[17] G. F. Du, Q. Z. Kong, F. H. Wu, J. B. Ruan, and G. B. Song, "An experimental feasibility study of pipeline corrosion pit detection using a piezoceramic time reversal mirror," Smart Materials and Structures, vol. 25, no. 3, Article ID 037002, 2016.

[18] G. Du, Q. Kong, H. Zhou, and H. Gu, "Multiple cracks detection in pipeline using damage index matrix based on piezoceramic transducer-enabled stress wave propagation," Sensors, vol. 17, no. 8, p. 1812, 2017.

[19] F. R. Wang, L. S. Huo, and G. B. Song, "A piezoelectric active sensing method for quantitative monitoring of bolt loosening using energy dissipation caused by tangential damping based on the fractal contact theory," Smart Materials and Structures, vol. 27, no. 1, Article ID 015023, 2018.

[20] G. Song, W. Li, B. Wang, and S. Ho, "A review of rock bolt monitoring using smart sensors,” Sensors, vol. 17, no. 4, p. 776, 2017.

[21] J. Wu, W. Li, and Q. Feng, "Electro-mechanical impedance (EMI) based interlayer slide detection using piezoceramic smart aggregates-A feasibility study," Sensors, vol. 18, no. 10, p. 3524, 2018.

[22] J. Wu, Q. Kong, W. Li, I. lim, and G. Song, "Interlayer slide detection using piezoceramic smart aggregates based on active sensing approach," IEEE Sensors Journal, vol. 17, no. 19, pp. 6160-6166, 2017.

[23] J. Zhang, Y. Huang, and Y. Zheng, "A feasibility study on timber damage detection using piezoceramic-transducer-enabled active sensing," Sensors, vol. 18, no. 5, pp. 1563-1574, 2018.

[24] J. Zhang, Y. Li, Y. Huang, J. Jiang, and S.-C. Ho, “A feasibility study on timber moisture monitoring using piezoceramic transducer-enabled active sensing," Sensors, vol. 18, no. 9, pp. 3100-3112, 2018.

[25] Z. Zhao, P. C. Chen, E. Zhang, and G. Y. Lu, "Health monitoring of bolt looseness in timber structures using PZTenabled time-reversal method," Journal of Sensors, vol. 2019, pp. 1-8, 2019.

[26] F. Han, J. Jiang, K. Xu, and N. Wang, "Damage detection of common timber connections using piezoceramic transducers and active sensing," Sensors, vol. 19, no. 11, p. 2486, 2019.

[27] J. Xu, J. Dong, H. Li, C. Zhang, and S. C. Ho, "Looseness monitoring of bolted spherical joint connection using electromechanical impedance technique and BP neural networks," Sensors, vol. 19, no. 8, pp. 1906-1924, 2019.

[28] B. Wang, Y. Sun, Y. Li, and C. Zhang, "Debonding damage detection in CFRP plate-strengthened steel beam using electromechanical impedance technique," Sensors, vol. 19, no. 10, pp. 2296-2308, 2019.

[29] C. Liang, F. P. Sun, and C. A. Rogers, "An impedance method for dynamic analysis of active material systems," Journal of Vibration and Acoustics, vol. 116, no. 1, pp. 120-128, 1994.

[30] Y. Yang, Y. Hu, and Y. Lu, "Sensitivity of PZT impedance sensors for damage detection of concrete structures," Sensors, vol. 8, no. 1, pp. 327-346, 2008. 
[31] S. Park, C.-B. Yun, Y. Roh, and J.-J. Lee, "Health monitoring of steel structures using impedance of thickness modes at PZT patches," Smart Structures and Systems, vol. 1, no. 4, pp. 339-353, 2005.

[32] S. Park, J.-W. Kim, C. Lee, and S.-K. Park, "Impedance-based wireless debonding condition monitoring of CFRP laminated concrete structures," NDT \& E International \& E International, vol. 44, no. 2, pp. 232-238, 2011.

[33] S. W. Shin and T. K. Oh, "Application of electro-mechanical impedance sensing technique for online monitoring of strength development in concrete using smart PZT patches," Construction and Building Materials, vol. 23, no. 2, pp. 1185-1188, 2009.

[34] D. Wang and H. Zhu, "Monitoring of the strength gain of concrete using embedded PZT impedance transducer," Construction and Building Materials, vol. 25, no. 9, pp. 3703-3708, 2011.

[35] C. P. Providakis, E. V. Liarakos, and E. Kampianakis, "Nondestructive wireless monitoring of early-age concrete strength gain using an innovative electromechanical impedance sensing system," Smart Materials Research, vol. 2013, no. 1, pp. 1-10, 2013.

[36] X. Hu, H. Zhu, and D. Wang, "A study of concrete slab damage detection based on the electromechanical impedance method," Sensors, vol. 14, no. 10, pp. 19897-19909, 2014.

[37] V. Annamdas, Y. Yang, and C. Soh, "Impedance based concrete monitoring using embedded PZT sensors," International Journal of Civil and Structural Engineering, vol. 1, no. 1, pp. 414-424, 2010.

[38] D. Wang, Q. Wang, H. Wang, and H. Zhu, "Experimental study on damage detection in timber specimens based on an electromechanical impedance technique and RMSD-based mahalanobis distance," Sensors, vol. 16, no. 10, p. 1765, 2016.

[39] L. Huo, F. Wang, H. Li, and G. Song, "A fractal contact theory based model for bolted connection looseness monitoring using piezoceramic transducers," Smart Materials and Structures, vol. 26, no. 10, p. 104010, 2017.

[40] G. Park and D. J. Inman, "Impedance-based Structural health monitoring," in Damage Prognosis: For Aerospace, Civil and Mechanical System, pp. 275-292, Wiley, Hoboken, NJ, USA, 2005. 